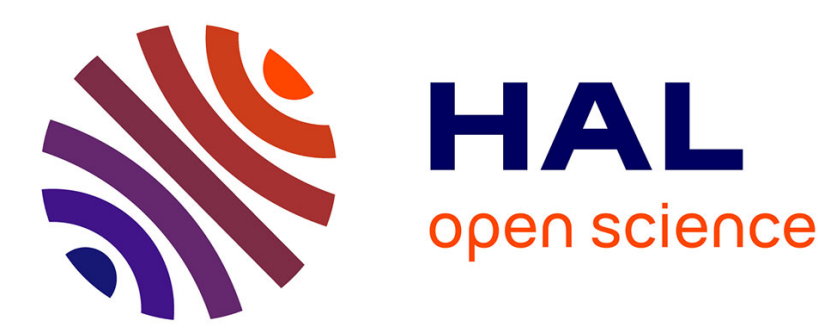

\title{
The catastrophic development of shear localization in thermoviscoplastic materials
}

F. Dinzart, C. Fressengeas, A. Molinari

\section{To cite this version:}

F. Dinzart, C. Fressengeas, A. Molinari. The catastrophic development of shear localization in thermoviscoplastic materials. Journal de Physique IV Proceedings, 1994, 04 (C8), pp.C8-435-C8-440. 10.1051/jp4:1994867 . jpa-00253428

\section{HAL Id: jpa-00253428 https://hal.science/jpa-00253428}

Submitted on 1 Jan 1994

HAL is a multi-disciplinary open access archive for the deposit and dissemination of scientific research documents, whether they are published or not. The documents may come from teaching and research institutions in France or abroad, or from public or private research centers.
L'archive ouverte pluridisciplinaire HAL, est destinée au dépôt et à la diffusion de documents scientifiques de niveau recherche, publiés ou non, émanant des établissements d'enseignement et de recherche français ou étrangers, des laboratoires publics ou privés. 


\title{
The catastrophic development of shear localization in thermoviscoplastic materials
}

\author{
F. Dinzart, C. Fressengeas and A. Molinari
}

Laboratoire de Physique et Mécanique des Matériaux, URA 1215 du CNRS, Université de Metz, Ile du Saulcy, 57045 Metz cedex 01, France

\begin{abstract}
résumé : Le couplage thermo-mécanique et l'adoucissement thermique sont les propriétés du matériau qui sont à l'origine du développement des bandes de cisaillement adiabatique. Cependant les défauts géométriques ou métallurgiques fournissent les sites où le processus de localisation de la déformation plastique est initié. Dans cet article, les effets de la forme et de l'amplitude des défauts sur la localisation de la déformation de cisaillement simple sont analysés à l'aide d'un modèle non linéaire : on formule des critères structurels de localisation asymptotique dans lesquels l'acuité des défauts module l'influence des facteurs rhéologiques, et l'on montre que, plus que leur amplitude, la forme des défauts permet d'interpréter la dispersion observée des déformations nominales à la rupture.
\end{abstract}

\begin{abstract}
Thermomechanical coupling and thermal softening are the critical material properties in the development of adiabatic shear bands. Besides the material effects, the sample geometrical imperfections and the material structural defects are regarded as providing the sites for the onset of the localization process. In this paper, the influence of the shape and size of the imperfections on the localization of the plastic flow in simple shear are analyzed within the framework of a nonlinear model: localization criteria are given in which the sharpness of the defects modulates the rheological effects. It is shown that, more than their size, the sharpness of the local imperfections may help to explain the observed scatter in the nominal failure deformation.
\end{abstract}

\section{INTRODUCTION.}

Thermomechanical coupling and thermal softening are at the origin of the localization of plastic flow in the neighborhood of sample geometrical imperfections or of material structural defects, and of the socalled adiabatic shear bands. It is well known that the onset of the localization process can be traced without ambiguity to the maximum in the shear stress $\tau$ vs. shear strain $\gamma$ diagram. For the same material under identical loading conditions however, a significant scatter in the nominal shear strain to failure is observed [1]. In the present paper, the observed scatter is ascribed to the shape and size of the imperfections in the local geometry or in the material structure, and to their effects on the localization process; the influences of the size and of the sharpness of the defects are analyzed within the framework of a one dimensional quasistatic and adiabatic model where elasticity is neglected, and localization criteria are provided in which the material parameters are modulated by the defects' sharpness.

\section{MODEL FORMULATION.}

Let us consider a slab of material (Fig. 1) of half height $h$ along the $(0, x)$ axis, of infinite extension along $(0, z)$, and of width $l(x)$ in the direction $(0, y)$. The slab is subjected to simple shear parallel to $(0, z)$. It is assumed that the only non zero particle velocity component is $u$ along the $(0, z)$ axis; in addition, one assumes that every considered quantity depends on the abscissa $x$ and on the time $t$ only. The problem thus defined is one-dimensional (1D); it is designed to model the torsion of a thin walled tube in a torsional Kolsky bar. 


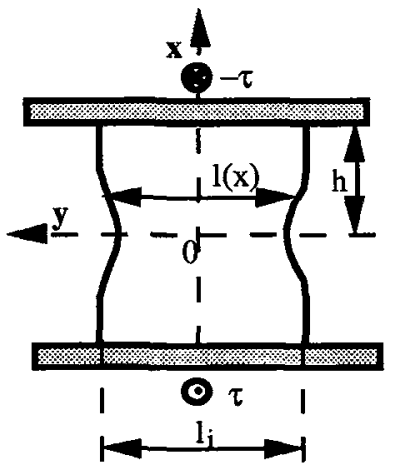

Fig. 1 : Specimen geometry and loading conditions.

Multidimensional features of shear band formation, such as the issue of their multiple nucleation and/or propagation around the specimen, are ignored in this model. Since the paper is focused on the imperfections analysis, the thermal diffusivity is (temporarily) neglected, as well as the inertial effects. Nevertheless, both are known to have a stabilizing influence, particularly in the final stages of the shear band development. reads

Inertial effects being neglected, the shear force does not depend on $\mathrm{x}$, and the equilibrium equation

$$
\forall \mathrm{x} \in[-\mathrm{h}, \mathrm{h}] \quad \tau(x, t) l(x)=\tau(0, t) l(0)
$$

In the assumed adiabatic conditions, the energy conservation is written as

$$
\rho c \dot{\theta}=\beta \tau \dot{\gamma}
$$

where $\rho$ denotes the mass density, c the massic thermal capacity, $\theta$ the temperature, $\dot{\gamma}=\frac{\partial u}{\partial x}$ the local strain rate and $\beta$ the Taylor-Quinney coupling constant, the value of which is roughly $\beta \cong 0.9$. Incompressibility of the material is assumed, and elastic deformations are not considered. The material behavior is assumed to be thermoviscoplastic, and its constitutive law is taken to be of the general form

$$
\tau=\mu(\mathrm{x}) \mathrm{F}(\gamma, \theta) \dot{\gamma}^{\mathrm{m}}
$$

where $\mathrm{m}$ is the strain rate sensitivity parameter. The shear modulus $\mu(x)$ is assumed to be $\mathrm{x}$-dependent in order to account for any material inhomogeneity or metallurgical defect. The coefficients used in the multiplicative form of the function $\mathrm{F}(\gamma, \theta)$ :

$$
\mathrm{F}(\gamma, \theta)=\theta^{v} \gamma^{\mathrm{n}}
$$

( $\mathrm{n}$ is the hardening coefficient and $v$ the thermal softening parameter) were identified by [1] for the CRS 1018 steel, as well as the strain rate sensitivity parameter $m(v=-0.38 ; n=0.015 ; m=0.019)$. Using the relations (3) and (4) allows integrating the energy equation (2) for either stress-controlled boundary conditions: $\tau(h, t)=\tau_{0}$, or under constant velocity boundary conditions: $\mathrm{u}(\mathrm{h}, \mathrm{t})=\mathrm{V}=\dot{\gamma}_{0} \mathrm{~h}$. Assuming that $\mathrm{m}$ is small enough, $\dot{\gamma}^{m}$ remains close to $\dot{\gamma}_{0}^{m}$ in a non uniform deformation; the temperature $\theta$ is then obtained as a function of the plastic deformation $\gamma$ and of the initial temperature $\theta_{0}$. Substituting $\theta(\gamma)$ in the relation (4) leads respectively to:

for stress controlled boundaries and

$$
F(\gamma, \theta(\gamma))=f(\gamma)=\theta_{o}{ }^{v}\left(1+a_{c} \gamma\right)^{v} \gamma^{n} \quad \text { with } \quad a_{c}=\frac{\beta \tau(x)}{\rho c \theta_{0}} \quad \text { and } \quad \tau(x)=\tau(0) \frac{l(0)}{l(x)}
$$

$$
F(\gamma, \theta(\gamma))=f(\gamma)=\theta_{0}{ }^{\nu}\left(1+a_{v} \gamma^{n+1}\right)^{v /(I-v)} \gamma^{n} \quad \text { with } \quad a_{v}=\frac{\mu(x) \beta(1-v) \dot{\gamma}_{0}^{m}}{\rho c(1+n) \theta_{0}^{1-v}}
$$

for velocity boundary conditions. In either case, the inequality $v+n<0$ indicates that a maximum in the shear stress occurs, beyond which thermal softening is predominant. Alternatively, we use the constitutive relation $f(\gamma)$ introduced by [2] in

$$
\tau=\mu(x) f(\gamma) \dot{\gamma}^{\mathrm{m}}=\mu(x) \gamma\left(1+\frac{b}{n} \gamma^{2}\right)^{n-1} \dot{\gamma}^{\mathrm{m}}
$$

The coefficients $(b, n)$ were also identified by [1] in the case of the HY-100 steel $(b=8000, n=0.49)$. A 
maximum in the shear stress occurs when $2 n<1$; such an inequality reflects the predominance of the material softening in the long term, whatever its actual physical origin.

Using $f(\gamma)$ as defined by (5), (6) or (7) in (3), then substituting the shear stress $\tau$ in the equilibrium equation (1) leads, after integration, to the the equation :

$$
\forall \mathrm{x} \in[-\mathrm{h}, \mathrm{h}] \quad(\mu(x) l(x))^{1 / m} \int_{\gamma_{0}}^{\gamma(x)} f(\xi)^{1 / m} d \xi=(\mu(0)(0))^{1 / m} \int_{\gamma_{0}}^{\gamma(0)} f(\xi)^{1 / m} d \xi
$$

where the initial plastic deformation $\gamma_{0}$ is assumed to be uniform. It is stated that there is asymptotic $\mathrm{L}_{\infty}$ localization of the plastic deformation in the cross-section $x=0$ if the deformation becomes unbounded for $x=0$, while remaining finite for every other $x$ [3]. Therefore an asymptotic $L_{\infty}$ localization criterion at $x=0$ is obtained by writing that the r.h.s. integral in ( 8 ) exists when $\gamma(0)$ becomes unbounded:

$$
\int_{\gamma_{0}}^{\infty} f(\xi)^{1 / m} d \xi<\infty
$$

The results obtained from this analysis for the expressions (5), (6) and (7) of the shear stress are reported in Table (1). For example, under stress controlled boundary conditions, the $\mathrm{L}_{\infty}$ localization criterion is

$$
v+n+m<0
$$

It is seen that the $\mathrm{L}_{\infty}$ localization inequalities are material criteria: $(10)$ illustrates the competition between the localizing influence of the material thermal softening $(v<0)$ and the uniformization effects of the strain hardening $(n>0)$ and of the strain rate hardening $(m>0)$. Clearly these criteria do not involve any structural or geometrical characteristic linked to the sample imperfections.

\begin{tabular}{|lccc|}
\hline$\tau=\mu \mathrm{f}(\gamma) \dot{\gamma}^{\mathrm{m}}$ & $\underline{\text { Knowles (7) }}$ & $\underline{\text { Stress controlled b.c. (5) }}$ & Velocity controlled b. c.(6) \\
$\mathrm{f}(\gamma)$ & $\left.x 1+b / n \gamma^{2}\right)^{n-1}$ & $\left(1+a_{c} \gamma\right)^{v} \gamma^{n}$ & $\left(1+a_{y} \gamma^{n+1}\right)^{v / 1-v \gamma^{n}}$ \\
$p$ (relation (13)) & $r m /(1-2 n-m)$ & $r m /(-v-n-m)$ & $r m(1-v) /(-v-n-m(1-v))$ \\
Instability & $2 n<1$ & $v+n<0$ & $v+n<0$ \\
$L_{\infty}$ & $2 n+m<1$ & $v+n+m<0$ & $v+n+m(1-v)<0$ \\
$L_{\infty}, r$ & $2 n+m(1+r)<1$ & $v+n+m(1+r)<0$ & $v+n+m(1-v)(1+r)<0$ \\
\hline
\end{tabular}

Table 1 : Results.

In what follows, we intend to show that accounting for the morphology of the defects leads to structural localization criteria, the nature of which is both material and geometrical.

\section{LOCAL GEOMETRICAL AND STRUCTURAL EFFECTS.}

When using $\gamma(0)$ as the driving parameter, or else assuming that the $L_{\infty}$ localization criterion (9) is fulfilled, the relation (8) appears to be an implicit integral equation for the unknown $\mathcal{X}(x)$. Two different solution procedures are used in this section: one is based on local asymptotic expansions valid in the neighborhood of the defect, while the other is an exact analytical solution obtained by means of convergent series developments of the integrals involved in equation (8). In this aim, one needs to describe the sample geometry and material non uniformity, and to provide a detailed account of the imperfection's region.

\subsection{Local solution.}

Let us define the sample width $l(x)$ in a neighborhood of a geometrical defect by the local development:

$$
-x_{1} \leq x \leq x_{1} \quad l(x)=l(0)+l_{r}(0)|x|^{r}
$$

where $r>0$ is the "sharpness" of the defect. It is assumed that $r>1$ and that $l_{r}(0)$ is small enough, in order to ensure that the defect wavelength remain large enough; then a 1D analysis retains validity. Similar developments can be conducted alternatively for material defects, without restrictions on the specified sharpness: the local shear modulus $\mu$ is therefore given by:

$$
-x_{1} \leq x \leq x_{1} \quad \mu(x)=\mu(0)+\mu_{r}(0)|x|^{r} .
$$

When the sharpness $\mathrm{r}$ satisfies $\mathrm{r}<1$, "micro-defects" resulting from a material singularity are described.

Assume that the asymptotic $\mathrm{L}_{\infty}$ localization criterion is satisfied; the integrals involved in the equation (8) can be calculated by using equivalents valid if the plastic deformations $\gamma(0)$ and $\gamma(x)$ are large enough [4]; these conditions are fulfilled when $x$ is small enough. The first order development of the 
deformation $\gamma(x)$ turns out to be of the form

$$
\gamma(x)=\alpha x^{-p}
$$

The results in $p$ are provided in Table 1 for the expressions (5), (6) and (7) of the shear stress. It is now required in addition that the nominal shear strain be bounded throughout the localization process. From the integrability condition (14) of the integral of the local plastic deformation (13):

$$
\int_{0}^{x_{1}} \gamma(x) d x<\infty
$$

it is seen that this boundedness condition is satisfied if and only if $\mathrm{p}<1$ : the obtained inequalities are called Lo, r localization criteria (see Table 1); for example with the stress controlled boundary conditions (5), the criterion $\mathrm{L}_{\infty}, \mathrm{r}$ happens to be:

$$
v+n+m(1+r)<0
$$

The competition of the thermal softening and of the strain and strain rate hardening in the localization process appears to be modulated by the defect's sharpness. The $\mathrm{L}_{\infty, \mathrm{r}}$ localization criterion is more stringent for the localizing factor (the thermal softening) than the corresponding $L_{\infty}$ criterion (10), since it ensures the boundedness of the nominal shear strain for a defect of strictly positive sharpness $r$; boundedness is guaranteed by the $\mathrm{L}_{\infty}$ criterion for an infinitely sharp defect $(\mathrm{r} \rightarrow 0)$ only. The uniformization effects of the strain rate sensitivity are all the more effective as the imperfections become flatter ( $r$ increasing); they are better accounted for in the $\mathrm{L}_{\infty, \mathrm{r}}$ localization criterion.

Local developments do not account for the defect's size. Therefore we now turn to a global solution, valid for every $x$, to assess the relative influence of the shape and size of the defects on the nominal shear to failure.

\subsection{Global solution.}

An analytic complete solution of the implicit equation (8) is obtained from convergent series developments of the involved integrals, which we write in terms of the hypergeometric functions ${ }_{2} \mathrm{~F}_{1}[5]$. For example, under the velocity boundary conditions (6) and for a smooth geometrical defect $(r>1)$, one uses the hypergeometric functions defined in (16) and (17):

$$
\begin{aligned}
& \int_{\gamma(x)}^{\infty}\left((1+a \xi)^{v} \xi^{n}\right)^{1 / m} d \xi=a^{-(1+n / m)} \frac{-m}{v+n+m}\left(\frac{1}{1+a \gamma}\right)^{1+n / m}{ }_{2} F_{1}\left(-(v+n+m) / m,-n / m ;-(v+n) / m ; \frac{1}{1+a \gamma}\right) \\
& =F_{\infty}(\gamma(x)) \\
& \int_{0}^{\gamma(x)}\left((1+a \xi)^{v} \xi^{n}\right)^{1 / m} d \xi=a^{-(1+n / m)} \frac{m}{n+m}\left(\frac{1}{1+1 / a \gamma}\right)^{1+n / m}{ }_{2} F_{1}\left(1+n / m,(v+n+2 m) / m ;(n+2 m) / m ; \frac{1}{1+1 / a \gamma(17)}\right. \\
& =F_{p}(\gamma(x))
\end{aligned}
$$

In that case, the equation (8) is written in the form

$$
\frac{F_{p}(\gamma(0))-F_{p}(\gamma(x))}{F_{p}(\gamma(0))-F_{p}\left(\gamma_{0}\right)}=1-\left(\frac{l(x)}{l(0)}\right)^{-1 / m}
$$

or, when the deformation $\gamma_{(0)}$ is infinite:

$$
\frac{F_{\infty}(\gamma(x))}{F_{\infty}\left(\gamma_{0}\right)}=1-\left(\frac{l(x)}{l(0)}\right)^{-1 / m}
$$

The series are expanded up to the 16th term in order to obtain convergence, but the computation time and the results accuracy are still much better than those obtained from a numerical integration. The local development (11) and the profile of constant width $\mathrm{l}_{\mathrm{i}}$ near the specimen edges are matched by a continuously derivable polynomial function of degree 5 ; the relative size of the geometrical defect is then $\varepsilon=1-l(0) / l_{i}$. The sample profile is plotted in Fig. 2 and 3 for given $\varepsilon$ and $\mathrm{r}$ values, and for various sharpness values $\mathbf{r}$ (respectively amplitude values $\varepsilon$ ).

The distribution of the computed plastic deformation is plotted in Fig. 4 and 5 for various defects' sharpness and defects' size; it is seen that the deformation becomes increasingly localized as the defects' sharpness increases ( $\mathrm{r}$ decreasing). The corresponding shear stress vs. shear strain diagrams are plotted in Figs. 6 and 7; larger $r$ values lead to lower rates of the decline in stress. It can be seen that the defect's sharpness has as much influence on the nominal strain to failure as its size. The attention is now focused on the effects of the local geometry, as specified by $\varepsilon$ and $r$, on the nominal shear strain $\gamma_{c}$ to failure, 
which is obtained at the beginning of the sharp decline in the applied shear stress. The dependence of $\gamma_{c}$ on the amplitude parameter $\varepsilon$ as predicted by this analysis is shown in Fig. (8). A decrease in $\gamma_{c}$ is observed as $\varepsilon$ increases; $\gamma_{c}$ varies approximately as loge, as predicted by [3] and observed by Duffy [6] in his torsional Kolsky bar experiments on the CRS 1018 steel. A significant scatter in the experimental nominal shear strain to failure is also noted, even for constant material and loading conditions.

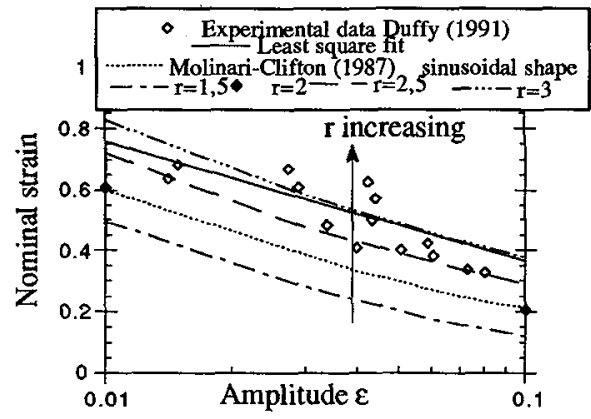

Fig 8: Dependence of the nominal shear strain to failure on the imperfection amplitude $\varepsilon$.

The sinusoiddal defect assumed by [3,6] is reproduced to the second order by using $r=2$ and $\operatorname{lr}(0)=4 \pi^{2} \varepsilon / h^{2}$; the nominal deformations to failure then merge with those obtained by Molinari-Clifton. However the predicted curve lies below the experimental data, and the actual strain to failure is underestimated. A variation of the sharpness roughly translates into a vertical shift of the representative plot, which allows to interpret the discrepancy: imperfections with sharpness parameters $r$ such that $2.5<\mathrm{r}<3$ provide predictions closer to the observed results. According to such an interpretation, the scatter in the observed values of the critical shear strain stems from the poor control which is exercised on the defects morphology.

Other effects are likely to be effective in this stage of the deformation localization, and may help understand the discrepancy between the critical strain predicted by Molinari and Clifton and those observed by Duffy, such as the delaying effects of heat conduction and inertia. However they cannot be used to explain the scatter in the observations.

\section{CONCLUSIONS.}

By assuming the deformation to be quasi-static and adiabatic, and by neglecting the elastic deformation, critical conditions for shear strain localization in simple shear have been given. These criteria account for the boundedness of the nominal shear strain; the detailed geometry of the sample is involved in the analysis, and the defect's sharpness modulates the material parameters in the obtained inequalities.

Comparisons of predictions of the model with experimental results for the critical strain to failure in a cold-rolled steel show that the detailed account of the specimen imperfections allows to obtain a good quantitative agreement. The sharpness and the size of the imperfections are shown to have a strong influence on the critical shear strain; therefore, the sample defects and the material structure need to be carefully controlled and characterized if the critical shear strain is to be used as a measure of the material resistance to failure.

\section{REFERENCES}

1) MARCHAND A. \& DUFFY J.; "An experimental study of the formation process of adiabatic shear bands in a structural steel", J. Mech. Phys. Solids., Vol. 36 n $^{\circ} 3$, (1988) 251-283.

2) KNOWLES J.K. ; "On finite antiplane shear for incompressible elastic materials", J. Austral. Math. Soc, Vol. 19B, (1976) 400-415.

3) MOLINARI A. \& CLIFTON R.J. ; "Analytical characterization of shear localization in thermoviscoplastic materials", J.of Appl.Mech. , Vol. 54, (1987) 806-812.

4) DIEUDONNÉ J. ; "Calcul infinitésimal", Hermann, (1968) pp. 84-87.

5) GRADSHTEYN I.S. \& RYZHIK I.M. ; "Table of integrals series and products", Academic press, New-York/USA, (1965) pp. 284-286.

6) DUFFY J. ; "Experimental studies of shear band formation through temperature measurements and high speed photography", J. Phys. IV Coll.C3, Suppl. J. Phys. III ,Vol.1, (1991) 646-652 
PLATE.

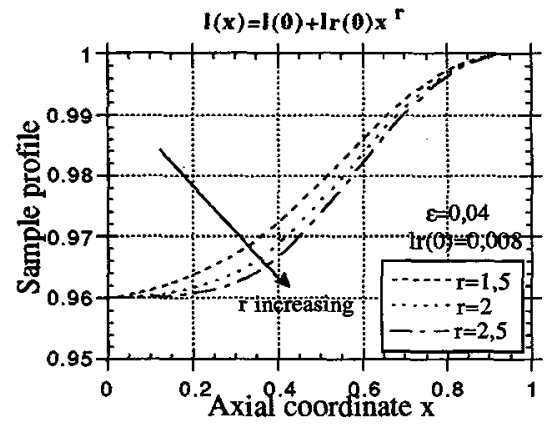

Fig. 2. - Specimen shape for various sharpness values.

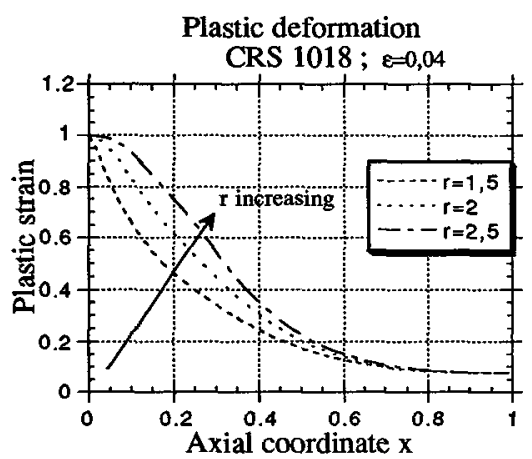

Fig. 4. - Plastic strain distribution for various sharpness values.

Plastic deformation

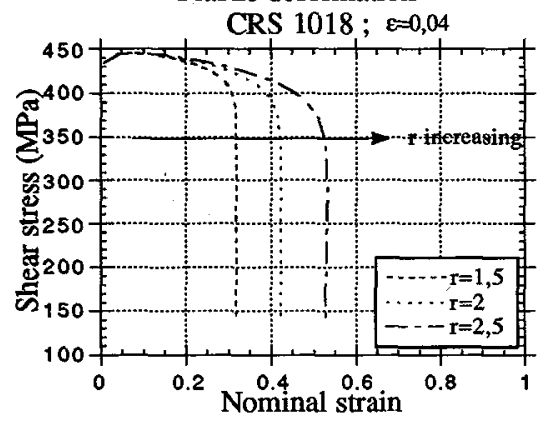

Fig. 6.- Nominal stress-strain curve for various sharpness values.

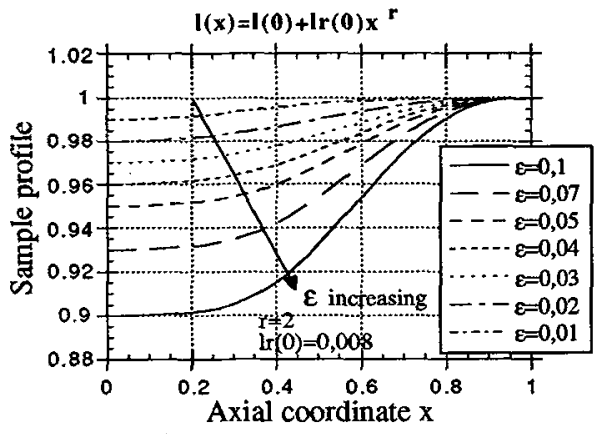

Fig. 3.- Specimen shape for various size values.

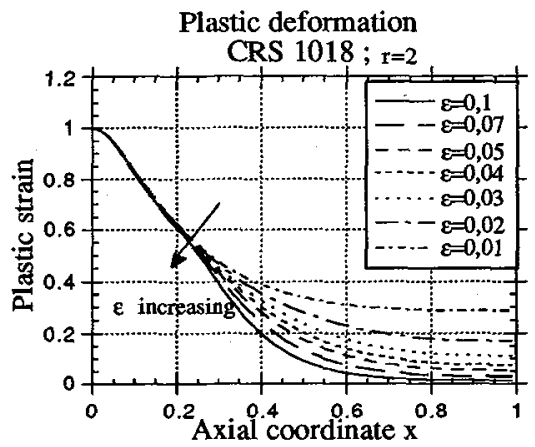

Fig. 5. - Plastic strain distribution for various size values.

Plastic deformation CRS $1018 \mathrm{r}=2$

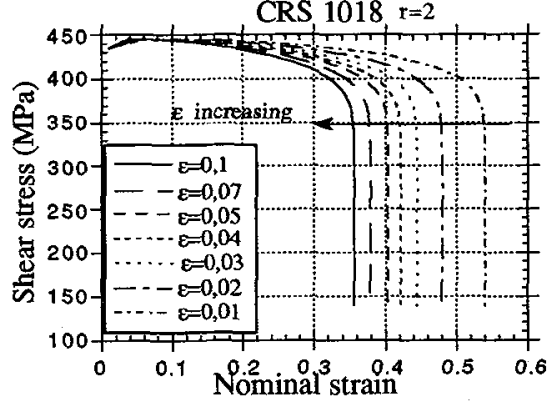

Fig. 7. - Nominal stress-strain curve for various size values. 